\title{
Application of S-Transform and Simultaneous Seismic Inversion to Interpret Bright Spots
}

\section{Nastaran Moosavi ${ }^{1 *}$ and Mohammad Mokhtari ${ }^{2}$}

${ }^{1}$ Department of Geophysics, Science and Research University, Tehran, Iran

${ }^{2}$ International Institute of Earthquake Engineering and Seismology, Tehran, Iran

\begin{abstract}
Bright spots are usually seen in seismic sections that can be interpreted as gas reservoirs. These local anomalies are identified as an increase in amplitudes on a seismic section. However, bright spots are not always gas indicators. They are also produced because of lithology changes. In this case, to prevent great costs of drilling, we need to understand the cause of bright spots. In this paper we use two methods which are mainly based on frequency and velocity analysis of seismic waves. Therefore, we apply simultaneous seismic inversion and S-transform on a 2D pre-stack seismic data which in its CDP gathers, several bright spots are seen. We just choose two of these bright spots arbitrarily. If they are due to hydrocarbon, P-wave velocity decreases sharply while S-wave velocity increases slightly, therefore decreases. Similarly, high frequencies being eliminated in the hydrocarbon areas. In contrast, if the bright spot is produced due to lithology change (gas saturated limestone/ limestone interface) then frequency and $\mathrm{P}$-wave velocity will increase while S-velocity decreases.
\end{abstract}

Keywords: S-Transform; Seismic; Reservoirs; Drilling; Gas; Energy

\section{Introduction}

Bright spots are abrupt increase in seismic amplitudes which result from large changes in acoustic impedance like gas sand/ shale boundary. But they can also be produced because of other phenomena, such as lithology change and tuning effects. Moreover, processing error, geometric or velocity focusing can be other reasons [1]. In last decades, geophysicists used post-stack seismic data, however bright spots are better seen in pre-stack seismic data than post-stack seismic data [2]. Therefore, we used a 2D line of pre-stack seismic data from a gas field in Persian Gulf, and applied simultaneous seismic inversion and S-transform to it. Simultaneous seismic inversion gives us three parameters of P-impedance, S-impedance, and density simultaneously. So, we can get information about P-velocity, S-velocity, and $\frac{\mathrm{V} p}{\mathrm{~V} s}$ ratio. The use of the ratio of compressional velocity to shear velocity is a good tool in identifying gas reservoirs. Presence of gas in the formation decreases the unit's velocity and density relative to the surrounding lithological units. This decrease produces a high-impedance contrast at the boundary where impedance changes resulting in a high-reflectivity zone or bright spot [3]. In addition, S-transform which transfers data from time domain to frequency domain provides us with frequency information. So, that, if the bright spot is produced because of hydrocarbon, high frequencies around the bright spot location will disappear. Although, if bright spot is due to lithology change (from soft to hard sediments), frequency increases in the bright spot area.

\section{Simultaneous seismic inversion}

For more than 3 decades, industry has known that shear seismic waves (S-waves) contain different rock information than do our standard compressional seismic waves ( $\mathrm{P}$-waves). Simultaneous seismic inversion is one of the methods which is used to invert pre-stack seismic data and the output of the inversion is three simultaneous parameters of acoustic impedance, shear impedance and density. From these parameters $\mathrm{V}_{\mathrm{p}}$ and $\mathrm{V}_{\mathrm{s}}$ are easily obtainable. Input data for this study includes fully processed NMO-corrected CDP gathers just before final stack and well information. First, seismic data will be transferred from offset domain to angle domain and three wavelets will be extracted after sorting the seismic data based on incidence angle to near, middle and far. Then we will estimate acoustic impedance, shear impedance and density via well log information. Three extracted wavelets will be used simultaneously in the equations from [4], which are approximations of Aki and Richards equations [5]. Inversion parameters are produced through equations which relate acoustic impedance, shear impedance and density to each other. By integration of pre-stack angle gathers, extracted wavelets and impedance models, we do inversion for the whole seismic profile [6]. The reflection coefficient or amplitude is given by the equation (1) which does not have any S-wave term, therefore, as a result the stack data does not have any information about S-wave velocity behavior. On the other hand, if reflection coefficients are evaluated for the individual rays corresponding to different source-receiver offsets, then the formulas are given in equation (2) [7]. These reflection coefficients now contain S-wave term. Thus, prestack amplitudes contain S-wave velocity information, which may be exploited to differentiate between true bright spots (hydrocarbon) and false bright spots (lithological changes).

$$
\begin{aligned}
& \mathrm{R}\left(\Phi_{1}\right)=\frac{\rho 2 V p 2-\rho 1 V p 1}{\rho 2 V p 2+\rho 1 V p 1} \Phi_{1}=0 \\
& \mathrm{R}(\theta)=\frac{1}{2}\left(\frac{\Delta v p}{v}+\frac{\Delta \rho}{\rho}\right)+\left[\frac{1}{2} \frac{\Delta V p}{V p}-2\left(\frac{V s}{V p}\right)^{2}\left(2 \frac{\Delta V s}{V s}+\frac{\Delta \rho}{\rho}\right)\right] \sin ^{2} \theta+\left(\frac{1}{2} \frac{\Delta V p}{V p}\right) \sin ^{2} \theta \tan ^{2} \theta
\end{aligned}
$$

where $R(\theta)$ is the reflection coefficient, $\theta=$ angle of incidence; $V p$ $=\mathrm{P}$-wave velocity in medium; $\Delta \mathrm{Vp}=\mathrm{P}$-wave velocity contrast across interface; Vs $=\mathrm{S}$-wave velocity in medium; $\Delta \mathrm{Vs}=\mathrm{S}$-wave velocity contrast across interface; $\rho=$ density in medium; $\Delta=$ density contrast across interface.

"Corresponding author: Nastaran Moosavi, Department of Geophysics, Science and Research University, Tehran, Iran, Tel: 44865179-82; E-mail: nastaran.moosavi@gmail.com

Received November 10, 2016; Accepted December 26, 2016; Published December 30, 2016

Citation: Moosavi N, Mokhtari M (2016) Application of S-Transform and Simultaneous Seismic Inversion to Interpret Bright Spots. J Pet Environ Biotechnol 7: 311. doi: 10.4172/2157-7463.1000311

Copyright: @ 2016 Moosavi N, et al. This is an open-access article distributed under the terms of the Creative Commons Attribution License, which permits unrestricted use, distribution, and reproduction in any medium, provided the original author and source are credited. 
Shear velocity in a formation depends on the formation's shear modulus and density while compressional wave velocity depends on shear moduli, bulk moduli and density. In most of lithological changes, if there is a change in P-wave velocity and density, there will be a corresponding change in $\mathrm{S}$-wave velocity.

$$
\begin{aligned}
& \mathrm{V}_{\mathrm{p}}=\sqrt{(k+4 / 3 \mu) / \rho} \\
& \mathrm{V}_{s}=\sqrt{\mu / \rho}
\end{aligned}
$$

Which $\mathrm{k}$ and $\mu$ are bulk and shear moduli respectively and $\rho$ is density.

It is observed from seismic data that velocities generally increase with depth. Densities also increase with depth so it must be that the bulk and shear moduli increase faster than the density. When seismic waves enter a gas area, density and compressional velocity both decrease. It can be concluded that elastic modules have more effect on velocity than density. Bulk moduli are the inverse of compressibility, as a result we expect bulk modules and compressional velocity drop suddenly in presence of gas. For shear velocity (which the determining factors are shear moduli and density) in gas zones, the factor of density has more influence on shear velocity while shear moduli remains almost unchanged. In this case, shear velocity must increase in gas. Dense rocks tend to have higher velocity. This signifies that the effect of elasticity on velocity is much greater than the effect of density. In porous rocks, depending on what type of fluid is located in the pores of the rock, the values of compressional and shear velocities are susceptible to change. Although, compressional velocity is relatively more sensitive to fluid type than shear velocity [8]. Therefore, combining $S$-wave and $P$-wave data as ratio will help in fluid type identification especially gas reservoirs.

\section{S-Transform}

We attempt to implement spectral decomposition method via S-transform to identify the responses of gas-saturated zones in the time-frequency domain. Gas and oil will absorb the high frequency energy and show strong low frequency energy [9]. When frequency changes (even it decreases, disappears or increases), it is much easier to see all these changes in frequency domain than in seismic section. As a result, we apply S-transform to the data to see how frequency reactions to different types of bright spots.

Hydrocarbon zones absorb high frequencies. But, what happens to seismic waves when they are trapped by hydrocarbon zones and lose their higher frequencies? To answer this question; one of the consequences of the relationship of stress to the product of particle velocity and impedance, is that for the same initiating stress or pressure, a seismic wave will have a lower particle velocity in a high impedance material than a low impedance material. As the low impedance material particle must travel further per frequency cycle (i.e. faster) than the high impedance material particle, so it will suffer more energy loss. This gives rise to the common observation that seismic waves suffer less high frequency attenuation in high impedance materials such as steel or water when compared to low impedance material such as wood or air.

There are different methods which can transfer the data into frequency domain such as S Transform, Short Time Fourier Transform (STFT), Gabor Transform (GT), Continuous Wavelet Transform (CWT). But among all of them we choose S-transform to transfer the seismic data to frequency domain and to evaluate the results. We illustrate several reasons why we use $\mathrm{S}$-transform for frequency analyze.
One of the pitfalls of the STFT is that STFT consists of a constant window width. A wide window gives better frequency resolution (frequency components close together can be separated) but poor time resolution (the time at which frequencies change). A narrower window gives good time resolution but poor frequency resolution. GT is a special case of the short-time Fourier transform that the windows size is a Gaussian function; $\exp \left[-\pi(\tau-t)^{2}\right]$. The continuous wavelet transform was developed as an alternative approach to the short time Fourier transform to overcome the resolution problem.

The wavelet analysis is done in a similar way to the STFT analysis, in the sense that the signal is multiplied with a function, wavelet, similar to the window function in the STFT, and the transform is computed separately for different segments of the time domain signal.

$S$ transform combines the merits of fourier transform and wavelet transform. It is an extension of continuous wavelet transform with Morlet wavelet as the basic wavelet. Here is the formula for wavelet transform (Wikipedia):

$$
\mathrm{X}(\mathrm{a}, \mathrm{b})=\frac{1}{\sqrt{a}} \int_{-\infty}^{+\infty} \Psi\left(\frac{t-b}{a}\right) x(\mathrm{t}) \mathrm{dt}
$$

$\Psi \quad$ is the mother wavelet, the shape or choice of this depends on the properties of the signal we wish to analyze, (b) is time and (a) is scale which is the inverse of frequency. The Morlet wavelet can be written in a simpler form as Addison [10]:

$$
\Psi(\mathrm{t})=\frac{1}{\sqrt[4]{\pi}} \exp \left[\mathrm{i} 2 \pi \mathrm{f}_{0} \mathrm{t}\right] \exp \left(-\mathrm{t}^{2} / 2\right)
$$

That $\frac{1}{\sqrt[4]{\pi}}$ is normalization factor, exp [i2 $\left.\pi \mathrm{f} 0 \mathrm{t}\right]$ is complex sinusoid and $\exp \exp \left[-\left(\frac{t}{2}\right)^{2}\right]$ is Gaussian bell curve.

This wavelet is simply a complex wave within a Gaussian envelope. The complex sinusoidal waveform is contained in the term $\exp \left[\mathrm{i} 2 \pi \mathrm{f}_{0} \mathrm{t}\right]$ $=\cos \left(2 \pi f_{0} t\right)+i \sin \left(2 \pi f_{0} t\right)$. The Gaussian envelope $\exp \exp \left[-\left(\frac{t}{2}\right)^{2}\right]$ confines the complex sinusoidal waveform. Figure below shows the real and imaginary parts of the Morlet wavelet together with its confining Gaussian envelope. The $\sqrt[4]{\pi}$ term is a normalization factor which ensures that the wavelet has unit energy (Figure 1).

Here is the formula for S-transform Chang [11]:

$$
\mathrm{S}(\mathrm{t}, \mathrm{f})=\int_{-\infty}^{+\infty} \mathrm{h}(t) \frac{|f|}{\sqrt{2 \pi}} \exp \left[-\frac{(\tau-t) 2 f^{2}}{2}\right] \exp [-\mathrm{i} 2 \pi f t] \mathrm{dt}
$$

With an easy substitution we come to this conclusion:

$$
\mathrm{S}(\mathrm{t}, \mathrm{f})=\exp [-\mathrm{i} 2 \pi f t] \mathrm{X}(\mathrm{a}, \mathrm{b})
$$

This means that $S$ transform is a phase correction of wavelet transform.

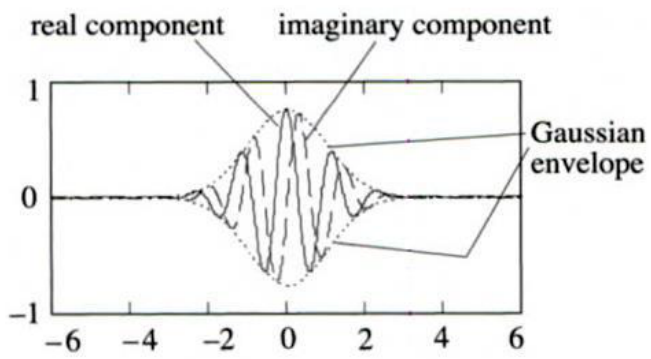

Figure 1: The real and imaginary parts of the Morlet wavelet together with its confining Gaussian envelope (Addison, 2002). 
Citation: Moosavi N, Mokhtari M (2016) Application of S-Transform and Simultaneous Seismic Inversion to Interpret Bright Spots. J Pet Environ Biotechnol 7: 311. doi: 10.4172/2157-7463.1000311

But the advantage of $\mathrm{S}$ transform to wavelet transform is that, $S$ transform is directly related to Fourier transform and provides resolution based on frequency instead of scale.

A problem with CWT is conversion of scale to frequency to make the interpretation easier but this takes a computer time. Morlet wavelet does not have this problem seriously but $S$ transform solve the problem completely because it achieved the Fourier frequency directly. Both (CWT and S transform) have a similar time-frequency resolution but different properties in their achievement. $S$ transform includes phase factor and retains absolute phase feature of every frequency. $\mathrm{S}$ transform has no phase shift and possess better time-frequency distribution which reflect abrupt changes of signals more directly and clearly [12]. Moreover, the window function for S-Transform is a function of $\mathrm{f}$. With a window function proportional to frequency, $\mathrm{S}$ Transform performs well in frequency domain analysis when the input frequency is low. When the input frequency is high, S-Transform has a better clarity in the time domain.

\section{Discussion}

A seismic CDP gather (CDP gather number 3332) is exhibited in Figure 2 which is from a $2 \mathrm{D}$ line of a $3 \mathrm{D}$ processed pre-stack seismic data. We are focusing on Fahlian formation as the study area which is a carbonate formation and is mainly made of limestone. Bright spots are seen easily in different parts of the gather. These bright spots can be hydrocarbon. On the other hand, they can be produced because of lithology change. The CDP gather which we are studying, includes several bright spots. We chose two of these bright spots arbitrarily to evaluate them whether they are due to hydrocarbon in this area or not. In this way, we get advantage of two geophysical methods; pre-stack seismic inversion and S-Transform. We have investigated the bright spots that have been observed whether they are gas indicators or they are because of lithology change.
After applying simultaneous inversion on pre-stack seismic data, compressional and shear velocities are obtained. At the location of bright spot (1) and (2), Shear and compressional velocities are illustrated in Figures 3 and 4. Density distribution is exhibited in Figure 5 , the amount of density increased from point (1) to pint (2). Based on the outputs of inversion, shear velocity has a higher value in point (1) than point (2), but compressional velocity in point (1) is less than point (2). According to explanations in last sections, this is mainly because of density reduction. However elastic moduli values had more effect on compressional velocity. With increase of density shear velocity increased while compressional velocity has a downward trend. As a result, $\frac{\mathrm{V} p}{\mathrm{~V}_{s}}$ ratio has a lower value in point (1) than point (2). In areas where gas exists, $\frac{\mathrm{V} p}{\mathrm{~V} s}$ ratio has dropped slightly (Figure 6).

Now we use spectral decomposition to see data in frequency domain. We loaded only the case study area in Matlab software (the program is prepared by the authors) to work easily with data. So the location of bright spots changes to CDP number 2832 and time between 1650 and $1800(\mathrm{~ms})$ to around 50 to $75(\mathrm{~ms})$ (Figure 7). Applying S transform on the seismic data shows that, frequency drops sharply at bright spot (1) while there is a spectacular increase in frequency amount of bright spot (2).

As a conclusion to what we see from the inversion and S-transform outputs is that; in point (1) density and compressional velocity decrease while shear velocity increases, we have increase in $\frac{\mathrm{V} p}{\mathrm{~V}_{s}}$ ratio, in contrast there is a reduction in frequency. On the other hand, in point (2); compressional velocity, density and $\frac{\mathrm{V} p}{\mathrm{~V}_{s}}$ ratio values are decreased while shear velocity and frequency have an increase in this

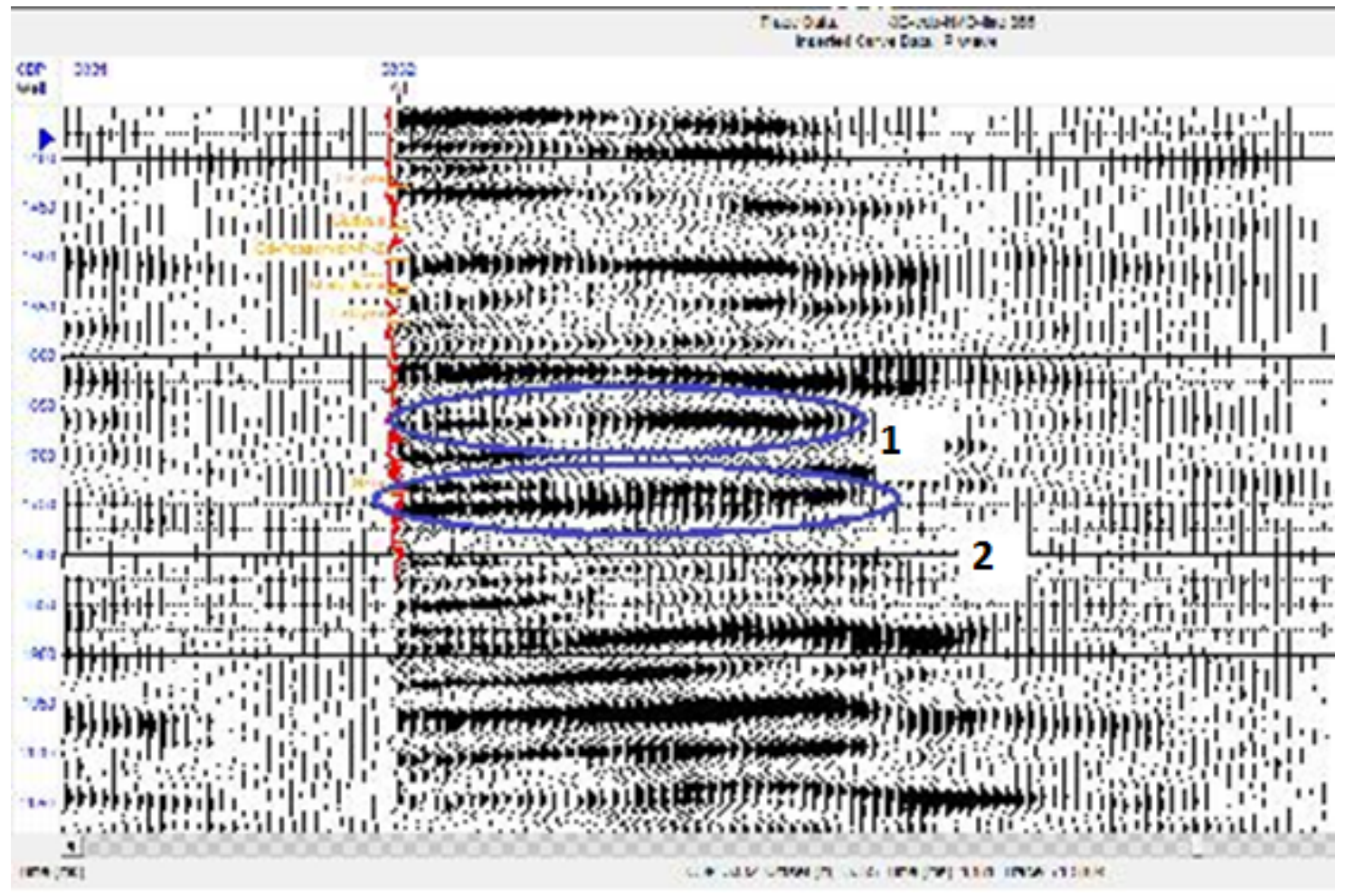

Figure 2: Two bright spots (1) and (2); CDP number 3332 
Citation: Moosavi N, Mokhtari M (2016) Application of S-Transform and Simultaneous Seismic Inversion to Interpret Bright Spots. J Pet Environ Biotechnol 7: 311. doi: 10.4172/2157-7463.1000311

Page 4 of 6

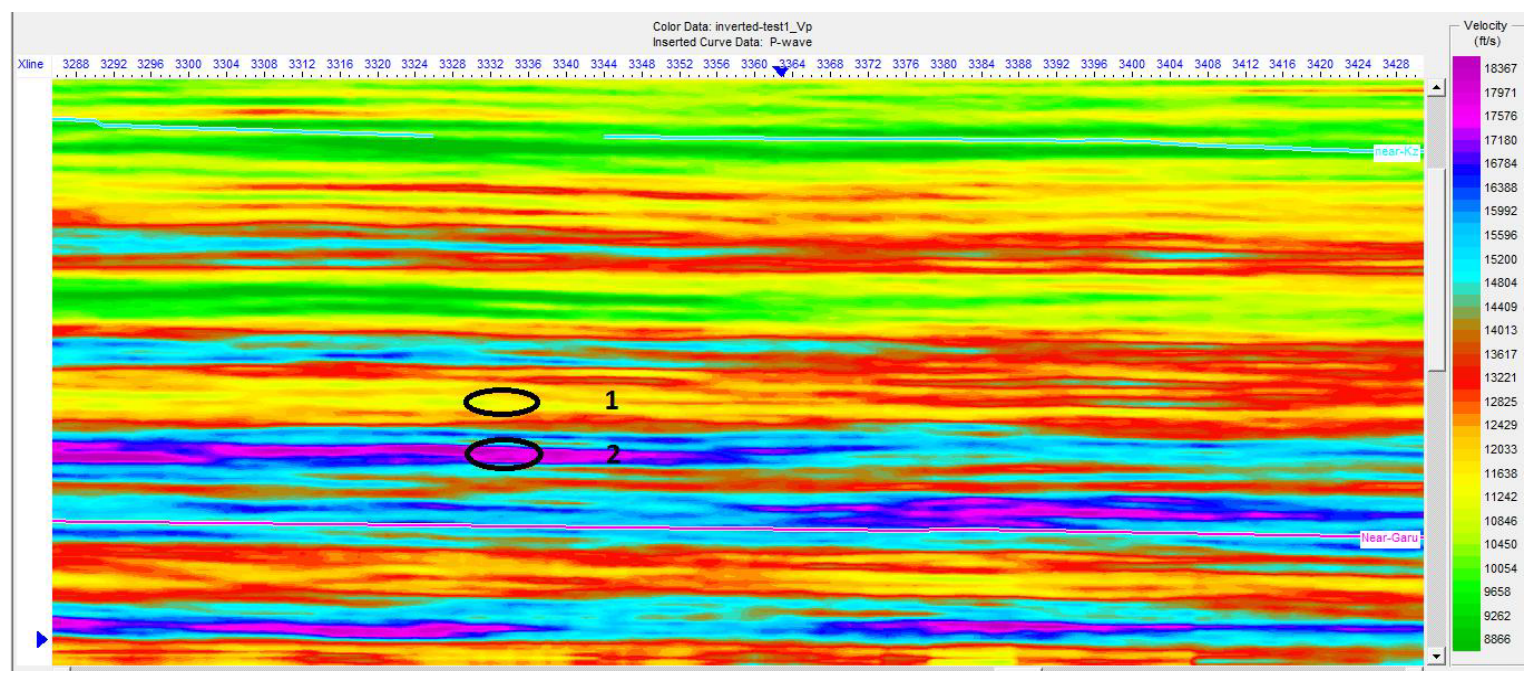

Figure 3: Vp obtained from inversion of pre-stack seismic data. $V p$ has increased from point 1 to point 2.

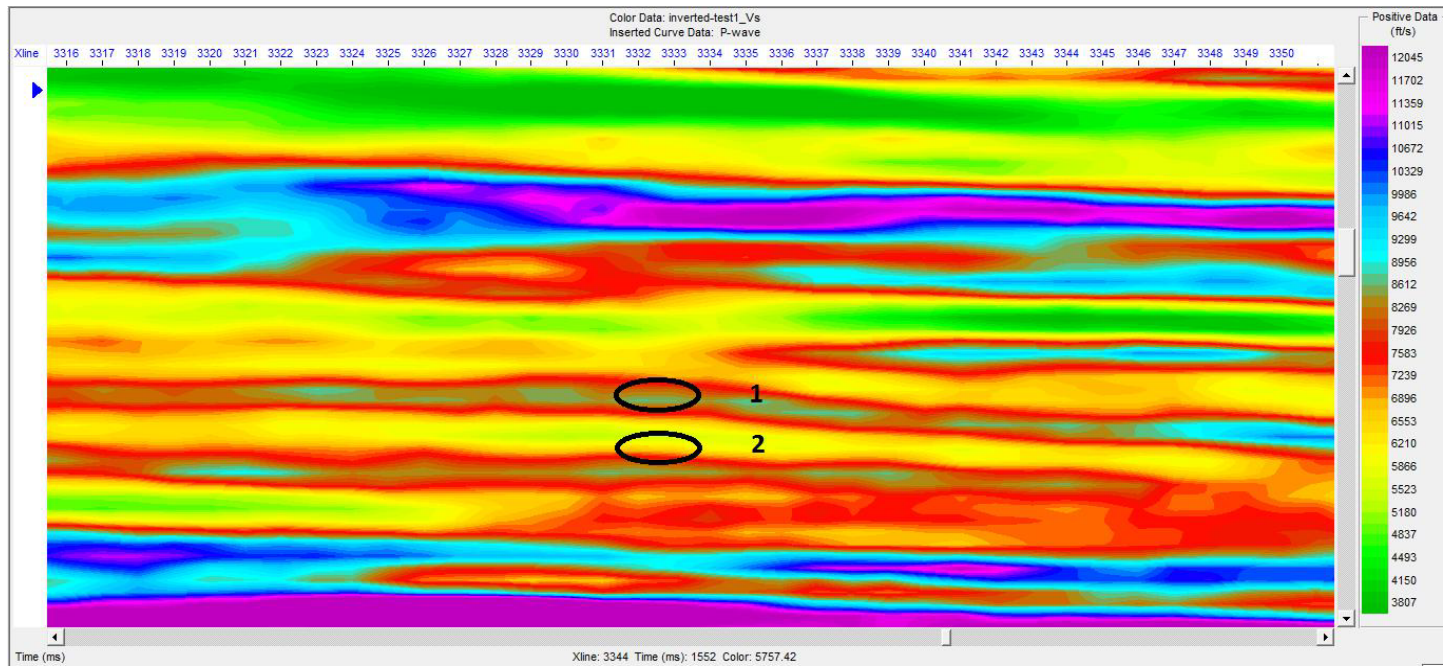

Figure 4: Vs obtained from inversion of pre-stack seismic data. Vs has decreased from point 1 to point 2.

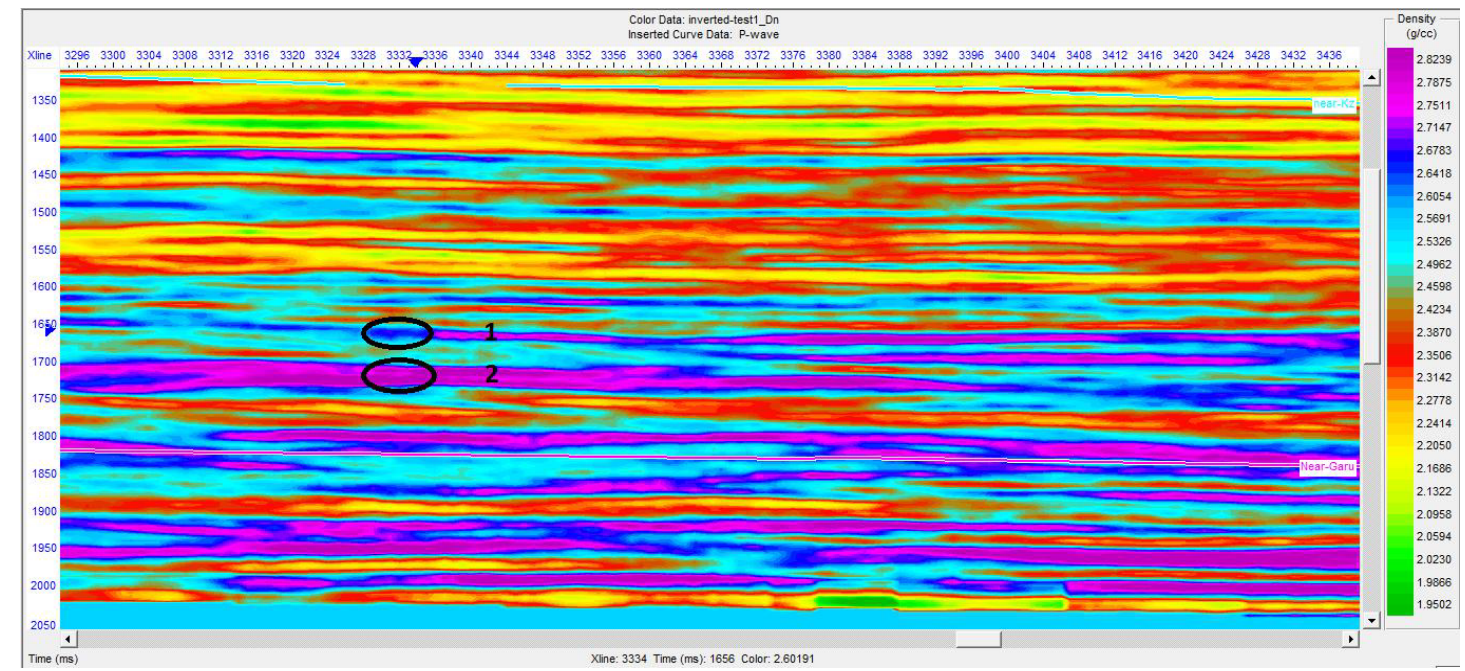

Figure 5: Density obtained from inversion of pre-stack seismic data. Density has increased from point 1 to point 2. 
Citation: Moosavi N, Mokhtari M (2016) Application of S-Transform and Simultaneous Seismic Inversion to Interpret Bright Spots. J Pet Environ Biotechnol 7: 311. doi: 10.4172/2157-7463.1000311

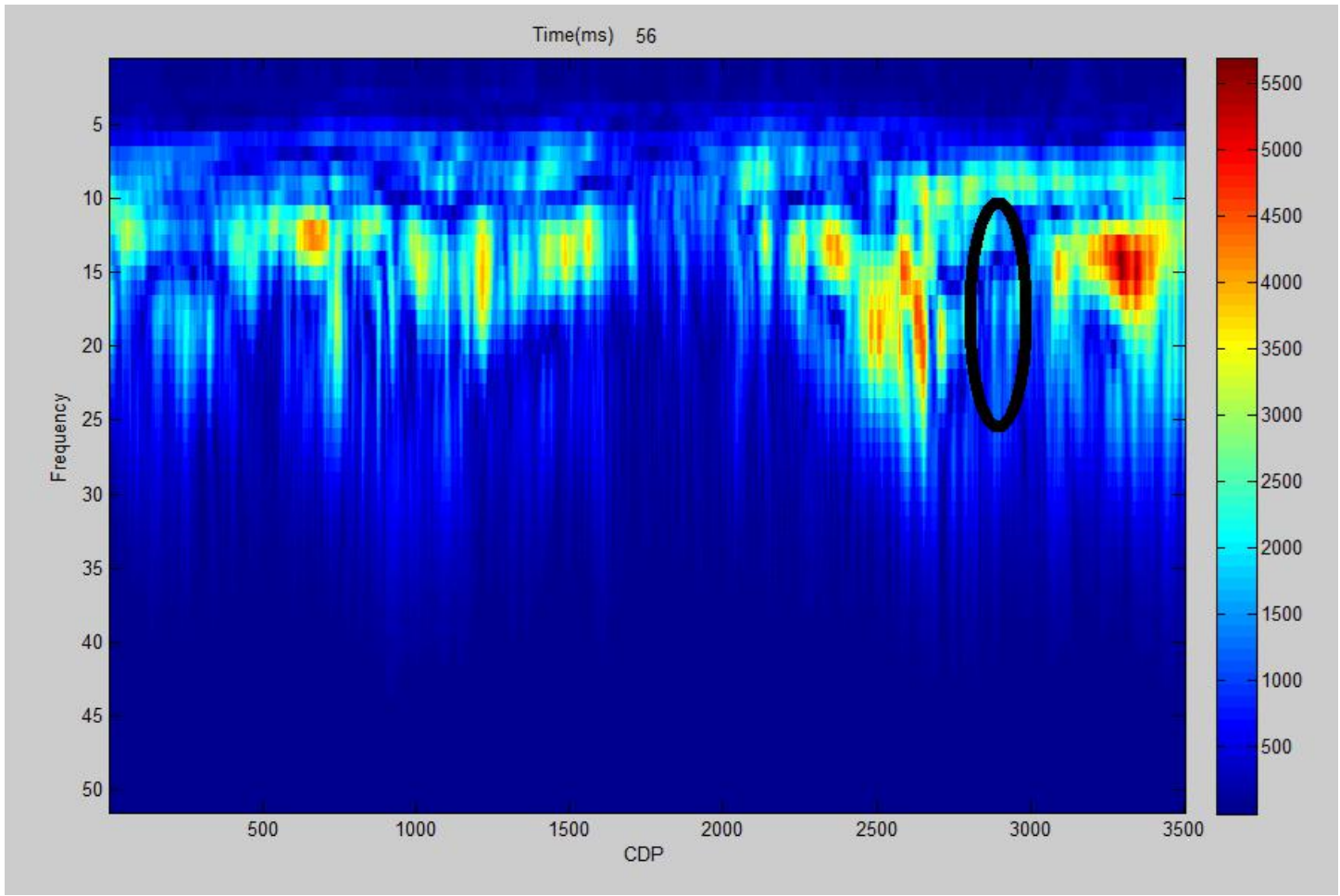

Figure 6: This is the ratio, has increased from point 1 to point 2.

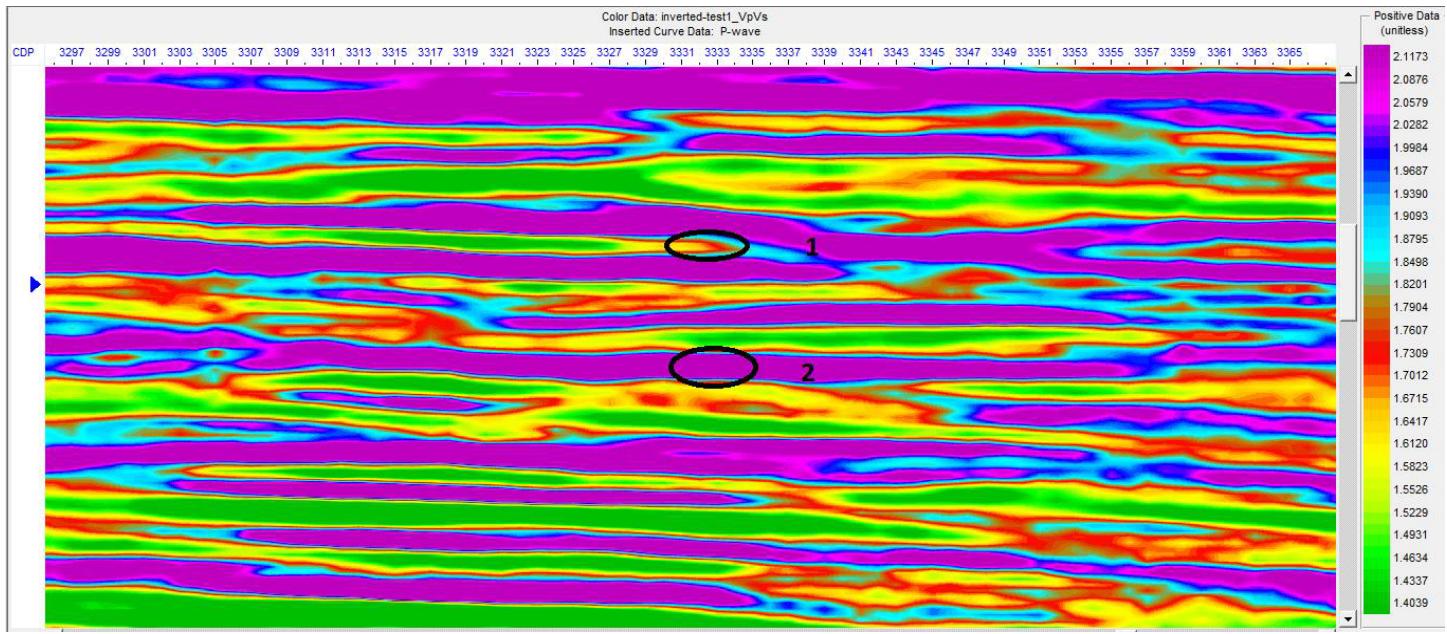

Figure 7: Frequency versus CDP number, when it comes to bright spot (1) frequency decreases dramatically.

point. All this information confirms that bright spot point (1) can be hydrocarbon accumulation, but bright spot point (2) should be resulted because of lithology change. In conclusion, we see if bright spot is due to lithology change, we can have slight changes of frequency; here we have even increase in frequency because there is a transfer from gas filled limestone to limestone. However, the presence of oil and gas causes the frequency drops sharply or even disappears completely in the reservoir area (Figure 8).

\section{Conclusion}

Bright spots are amplitude anomalies that can indicate the presence of hydrocarbons. They are produced because of different reasons such as processing problems, lateral velocity variations, lithology change and gas reservoirs. Obviously, the ultimate goal of geophysicists is to find hydrocarbon reservoirs. To overcome the risks of drilling, we applied simultaneous seismic inversion as well as S-transform on a 2D seismic section. The results show that when bright spots are due to hydrocarbon; density, compressional velocity and ratio dropped suddenly while shear velocity increases slightly. On the other hand, when bright spot is due to lithology change; density, compressional velocity and ratio increased spectacularly, however, shear velocity decreased mainly because of density reduction. In the gas zone, high frequency was absorbed by gas while in lithology change zone, frequency increased spectacularly because of carbonate formation. Overall, frequency and velocity are two powerful keys to differentiate hydrocarbon from lithology change when there are bright spots in seismic data. 
Citation: Moosavi N, Mokhtari M (2016) Application of S-Transform and Simultaneous Seismic Inversion to Interpret Bright Spots. J Pet Environ Biotechnol 7: 311. doi: 10.4172/2157-7463.1000311

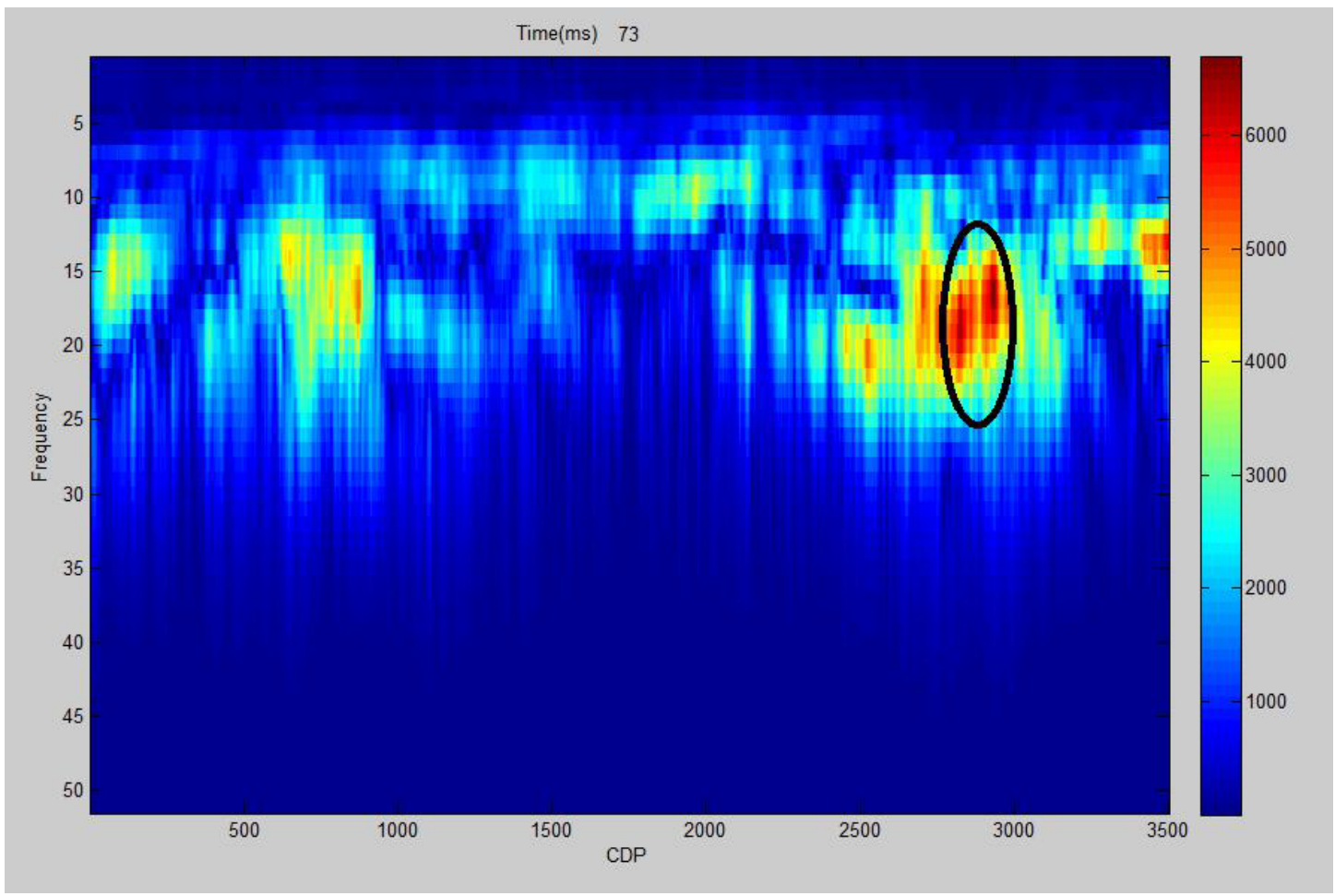

Figure 8: Frequency spectra at the location of bright spot (2); frequency has a high amount in this area.

\section{References}

1. Castagna J, Backus M (1993) Offset-dependent reflectivity, Theory and practice of AVO analysis: Society of Exploration Geophysics, Tulsa, OK.

2. Goodway B (2001) Avo and lame constants for rock parameterization and fluid detection. CSEG Recorder, p: 39-60.

3. Sengbush R (1983) Petroleum exploration; A quantitative introduction.

4. Fatti JL, Smith GC, Vail PJ, Strauss PJ, Levitt PR (1994) Detection of gas in sandstone reservoirs using AVO analysis: a 3D Seismic Case History Using the Geostack Technique: Geophysics 59: 1362-1376.

5. Aki K, Richards PG (2002) Quantitative Seismology, (2ndedn) W.H. Freeman and Company. New York.

6. Moosavi N, Mokhtari M (2016) Application of post-stack and pre-stack seismic inversion for prediction of hydrocarbon reservoirs in one of the gas fields, Persian Gulf: World Academy of Science, Engineering and Technology.
International Journal of Geological and Environmental Engineering.

7. Shuey RT (1985) A simplification of the Zoeppritz equations. Geophysics 50 609-614.

8. Cheng CH, Toksöz MN (1979) Inversion of seismic velocities for the pore aspect ratio spectrum of a rock. Journal of Geophysicsal research 84: 7533-7543.

9. Zabihi NE, Siahkoohi HR (2006) Single Frequency seismic attribute based on short time fourier transform, continuous wavelet transform and s-transform. 6th International Conference \& Exposition on Petroleum Geophysics, Kolkata.

10. Addison P (2002) The illustrated wavelet transform handbook: introductory theory and applications in science, engineering, medicine and finance. CRC Press.

11. Chang S (2009) An introduction to S-transform for time frequency analysis. Ashlee Copeland Publsihing.

12. Xiong C, Huang Y (2008) Intelligent robotics and applications: First International Conference, ICIRA 2008 Wuhan, China, October 15-17. 